\title{
Making Children Legitimate Die Gesetzgebung Justinians zu concubinatus und liberi naturales in Nov 74 und ihre Vorläufer
}

On the $14^{\text {th }}$ of June 538, the Emperor Justinian issued a constitution known as Novel 74 in the Corpus Iuris Civilis. The aim of this constitution was to determine all the possibilities for the legitimation of 'natural' children. In Novel 74 Justinian determined what the conditions are that allow children to be called either natural or legitimate as well as who the children are who are neither legitimate nor natural. After that, he created three mechanisms to legitimate children: by their parents' marriage, by imperial decision on demand of the father, or on the children's demand based on their father's testament. But is this legislation as new as Justinian pretends it to be?

Am 4. Juni 538 richtete Kaiser Justinian an den praefectus praetorio Orientis Johannes eine Konstitution, die als Novelle 74 geführt wird. ${ }^{1}$ Sie stellt sich selbst in eine Reihe mit früheren Konstitutionen Justinians und seiner Vorgänger (Justin),

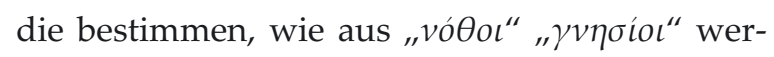
den: aus "unechten“/"unehelichen" Kindern „vollbürtige"/,eheliche“.

Schon die älteste lateinische Übersetzung, das Authenticum, erkennt darin die "filii naturales" einerseits, die "filii legitimi" andererseits, und präzisiert in der Rubrik den Inhalt der Novelle folgendermaßen:

"Quibus modis naturales fillii efficiuntur legitimi et sui, supra illos modos qui superioribus constitutionibus continentur."

„Auf welche Weisen natürliche Kinder zu legitimen Hauskindern werden, über jene Weisen

\footnotetext{
* Für Hilfe mit dem Deutschen danke ich herzlich Johannes Platschek.

${ }^{1}$ Dazu zuletzt Vigneron, Politique moralisatrice 583592; DERS., Novelle 74.5 729-737; LANATA, Figli della passione 487-493; TOMULESCU, Justinien et le concubinat 299-326.
}

hinaus, die in den älteren Konstitutionen enthalten sind. “2

Tatsächlich gibt Justinian einerseits eine Synthese der bereits bisher möglichen Wege der Legitimierung der filii/liberi naturales; er bekennt sich dabei zu den repressiven Regelungen seines Vorgängers Justin; gleichzeitig stellt er die eigenen Ergänzungen als Neuerungen ohne Vorläufer dar, die in besonderer Weise der Natur ( $\phi \dot{v} \sigma \iota \varsigma)$ und der Reinheit der Sitten ( $\sigma \omega \phi \rho \sigma \sigma v ́ v \eta)$ entsprächen.

Was verbirgt sich hinter diesen rhetorischen Anstrengungen?

\section{1. „Yvpíoı"/Liberi legitimi}

Gedanklicher Ausgangspunkt sind die liberi (oder filii) legitimi von Geburt. Sie stammen aus einer anerkannten Ehe (matrimonium iustum ${ }^{3}$ ),

\footnotetext{
${ }^{2}$ LUCHETTI, Legittimazione; FRIEDL, Konkubinat; TOMULESCU, Justinien et le concubinat 299f.

${ }^{3}$ VOLTERRA, Iniustum Matrimonium 441-470; GAUDEMET, Justum matrimonium 309-366; OLSEN, Femme et enfant $16 \mathrm{f}$.
} 
für die - neben möglichen Eheverboten ${ }^{4}$ - die innere Einstellung der Eheleute, die affectio maritalis von entscheidender Bedeutung ist. ${ }^{5}$ Dieser Ehewille konnte schon bisher insbesondere durch die Bestellung einer Mitgift (dos) und die Errichtung einer Eheurkunde (instrumentum nuptiale) bewiesen werden. ${ }^{6}$ Kinder aus einem iustum matrimonium muss der Vater als die seinen anerkennen, indem er sie "aufhebt" (tollere liberos ${ }^{7}$ ); er nimmt das Kind äußerlich erkennbar in die Familie auf.

Auch zu den filii/liberi legitimi von Geburt äußert sich Justinian in der Novelle. Er will Scheinehen, in denen sie geboren werden, vermieden wissen: Der Beweis des Ehewillens kann künftig nur noch in bestimmten Formen erbracht werden. In den höchsten Ständen ${ }^{8}$ muss dazu eine Mitgift entrichtet, das voreheliche Geschenk (, $\pi \rho \rho \gamma \alpha$ -

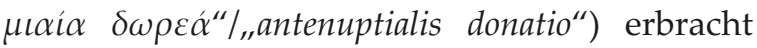
werden und "alles andere [geschehen], was sich für diese ehrenhafteren Stände geziemt ". ${ }^{\prime}$ Die mittleren Schichten ${ }^{10}$ müssen, wenn keine Mit-

\footnotetext{
${ }^{4}$ Nov 74, 1, pr; z.B. unter Verwandten und Verschwägerten, aufgrund des Ranges des Mannes, aufgrund des Glaubens etc.; KASER, Römisches Privatrecht 162168.

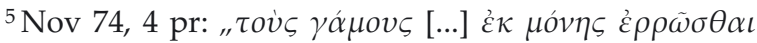

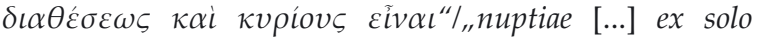
affectu valeant et ratae sint". URBANIK, Broken marriage promise 126f.; WOLFF, Doctrinal Trends 261-319.

${ }^{6} \mathrm{C}$ 5, 17, 11: "non enim dotibus, sed adfectu matrimonia contrahuntur."

${ }^{7}$ Dazu CAPOgrossi ColognesI, Tollere liberos 131146; SANTORO, Sul 'Tollere liberos' 273-278.

${ }^{8}$ Nov 74, 4, 1-2; namentlich Senatoren und hoch-

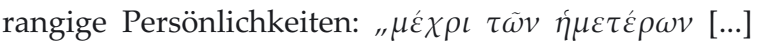
$\sigma v \gamma \kappa \lambda \eta \tau \iota \kappa \tilde{\omega} v \quad \kappa \alpha i \quad \tau \tilde{\omega} v \quad \mu \varepsilon \gamma \alpha \lambda$ о $\tau \rho \varepsilon \pi \varepsilon \sigma \tau \alpha \dot{\alpha} \omega \nu$

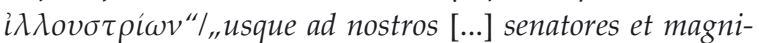
ficentissimos illustres"; VOCI, Novi studi 246f.; VIGNERON, La politique moralisatrice 584f; TOMULESCU, Justinien et le concubinat, 314 ; URBANIK, Broken marriage promise 123f.; WOLFF, Written and Unwritten Marriages $90 \mathrm{f}$.

${ }^{9}$ Nov 74, 4, 1; URBANIK, Broken marriage promise 132f.; VIGNERON, Politique moralisatrice 584f.

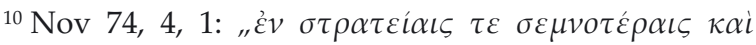

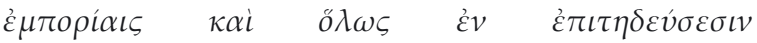

gift oder voreheliche Schenkung bescheinigt wird, eine Erklärung vor einem kirchlichen Amtsträger abgeben. ${ }^{11}$ Nur in den untersten

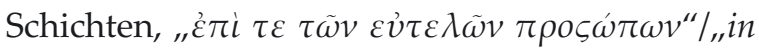
vilibus personis", verzichtet Justinian auf besondere Formen. ${ }^{12}$

Umgekehrt bekämpft Justinian die Berufung der Väter auf fehlende affectio und fehlende Ehe, wenn der Frau und Mutter die Ehe „unter Berührung der heiligen Schriften“ oder "unter Eid in den Gebetshäusern" versprochen wurde. ${ }^{13}$ Nachkommen aus einer solchen Verbindung sind "ehelich auch gegen den Willen des Vaters"

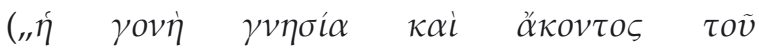
$\pi \alpha \tau \rho o ́ \varsigma^{\prime \prime} /$,soboles legitima etiam invito patre"), ${ }^{14}$ aufgrund der Dauer dieser Verbindung und des Eids, den der Vater abgelegt hat. ${ }^{15}$

\section{Die "vó $\theta o$ ı"/liberi naturales in Nov 74, 6}

"NóOoı"/liberi naturales sind nicht ehelich geborene Kinder im Sinne von Nov 74, 4-5: Die Eltern sind weder durch ein matrimonium iustum noch aufgrund eines feierlichen Eheversprechens verbunden. ${ }^{16}$

$\dot{\alpha} \xi \iota \lambda o \gamma \omega \tau \varepsilon \dot{\varepsilon} \rho \iota \varsigma^{\prime \prime} I_{\text {„In }}$ militiis honestioribus et negotiis et omnino professionibus dignioribus".

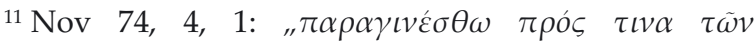

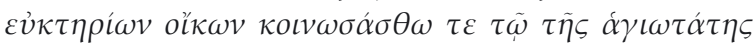
$\dot{\varepsilon} \kappa \varepsilon \dot{\imath} \eta\rceil \varsigma \dot{\varepsilon} \kappa \kappa \lambda \eta \sigma i \alpha \varsigma \dot{\varepsilon} \kappa \delta i \kappa \omega^{\prime \prime} /, v e n i a t$ ad quandam orationis domum et fateatur sanctissimae illius ecclesiae defenso$r i$.

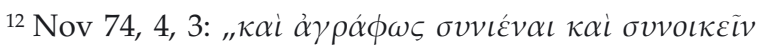
$\dot{\alpha} \lambda \lambda \eta \dot{\lambda}$ oıs"/,et non scripto convenire et matrimonia celebrare inter utrosque". URBANIK, Broken marriage promise 132f.; VignERON, Politique moralisatrice 584f.; TOMULESCU, Justinien et le concubinat 314.

${ }^{13}$ Nov 74, 5, pr; URBANIK, Broken marriage promise 132f.; VIGNERON, Politique moralisatrice 590f,.

${ }^{14}$ Nov 74, 5, 1.

${ }^{15}$ Vigneron, Politique moralisatrice 585f.; DERS. Novelle $74.5,732$ f.

16 WAELKENS, Enfants naturels 399f.; OLSEN, Femme et enfant 93f.; LUCHETTI, Legittimazione 1f.; VocI, Novi 
Filii / liberi naturales dürfen aber auch nicht aus verbotenen Verbindungen stammen, ${ }^{17}$ wie sie Justinian in Nov 12, 1 von 535 aufzählt, etwa aus inzestuöser Verbindung. ${ }^{18}$

Bereits aus älteren Konstitutionen ergibt sich, dass Kinder ohne bekannten Vater, so genannte spurii, ${ }^{19}$ nicht als liberi naturales gelten.

Im Sinne unserer Novelle handelt es sich nur um Kinder freier Eltern. ${ }^{20}$

Diese Voraussetzungen kennzeichnen die liberi naturales Justinians als Kinder aus einem concubinatus, ${ }^{21}$ das heißt aus einer dauerhaften Lebensgemeinschaft, bei der entweder die affectio

studi 219f.; NIZIOLEK, Legal effects of concubinage; DIES., Meaning of the Phrase 317-344; TOMULESCU, Justinien et le concubinat 302f.; JANEAU, Adrogation des liberi naturales.

17 Z.B. zwischen Juden und Christen: RABELLO, Problema dei matrimoni 213-224.

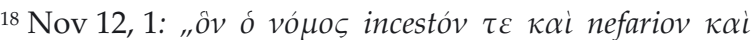
damnatov $\kappa \alpha \lambda \varepsilon \tilde{I}^{\prime \prime}$ " "quas lex incestas et nefandas et damnatas vocat". Sie dürfen nicht naturales genannt werden, und können nicht die clementia des Kaisers in An-

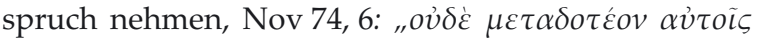

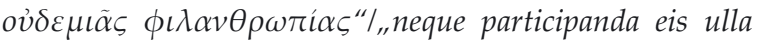
clementia est".

${ }^{19}$ Z.B. in Gai. inst. 1, 64; PePPE, Paelex e spurius 354f.; FRIEDL, Konkubinat 143f.; LANATA, Figli della passione 487f.; SANDIROCCO, Concubinato 226f. In C 6, 57, 5, 1 (529) verbot Justinian Zuwendungen einer Frau höheren Standes (illustris mulier) von Todes wegen oder inter vivos an einen "spurius, cui pater incertus sit", wenn daneben ein "filius ex iustis nuptiis" vorhanden ist.

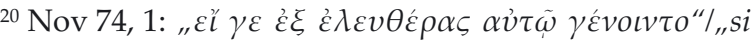
ex libera ei fiant".

${ }^{21}$ In C 6, 57, 5, 2 erscheinen die Kinder einer Konkubine als solche aus einer erlaubten Lebensgemeinschaft: "concubina liberae condicionis constituta filium vel filiam ex licita consuetudine ad hominem liberum habita procreaverit"; FAYER, Familia romana 1, 1-54 ; SANDIROCCO, Concubinato 197-230; OlsEN, Femme et enfant 53f, 154f.; GAUDEMET, Union libre 377-392; KARABELIAS, Pratique du concubinat 183-201; Rousselle, Concubinat et adultère 75-84; TOMULESCU, Justinien et le concubinat 316f.; WATSON, Law of Persons 1-10; DANIELI, Concubinato 175-179; SOLAZZI, Concubinato 269-277; Bonfante, Corso 1, 315f.; Plassard, Concubinat romain; MEYER, Römischer Konkubinat. maritalis fehlt oder eine Ehe aus Standesgründen nicht möglich ist.22 Die Geschlechtsgemeinschaft darf aber nicht wegen Inzests, Ehebruchs oder aus Altersgründen verboten sein. ${ }^{23}$

Im Zentrum der Novelle 74 steht die nachträgliche Legitimierung von Kindern aus einer solchen Verbindung. ${ }^{24}$

\section{Die Legitimierung von liberi naturales durch nachfolgende Ehe: Nov 74 Praefatio}

Sie kann durch nachträgliche Ehe der Eltern erfolgen. ${ }^{25}$ Justinian nimmt auf hergebrachtes Recht und eigene frühere Konstitutionen in diesem Bereich Bezug, ${ }^{26}$ denen er in korrigierter und präzisierter Form dauerhafte Geltung verschaffen will.

Danach gilt Folgendes:

- Eine nachfolgende Legitimierung durch Ehe durch Aufkommen der affectio maritalis ist nur

${ }^{22}$ Z.B. Plaut., Trinummus 691.

23 D 25, 7, 3, 1 (Marc. 12 inst.); D 25, 7, 4 (Paul. 19 respon.); D 23, 2, 56 (Ulp. 3 disp.): kein "incestum“; D 25, 7, 1, 3 (Ulp. 2 ad. leg. Iul. et Pap.): keine Verwandtschaft - "quia prope nefaria est huiusmodi coniunctio, et ideo huiusmodi facinus prohibendum est"; D 25, 7, 1, 4: keine Unmündige unter zwölf Jahren "Cuiuscumque aetatis concubinam habere posse palam est nisi minor annis duodecim sit"; Paul. Sent. 2, 20, 1; kein Ehebruch; GAUDEMET, Droit romain et principes canoniques 173-196.

${ }^{24}$ VignERON, Politique moralisatrice 588f.

${ }^{25}$ FAYER, Familia romana 3, 45f.; LUCHETTI, Legittimazione 173-288; Migliorini, Adozione 345f.; NizIOLEK, Legal effects of concubinage 84-86; VAN DE WIEL, Légitimation par mariage subséquent 307-350; BONFANTE, Corso 1, 372-374; DESPIAU, Enfant né du concubinatus 65-71; Le DeufF, Du Concubinatus 75-78; MENARD, Concubinat et enfants naturels 53-55; MEYER, Römischer Konkubinat 143f., 149f.

${ }^{26} \mathrm{C} 5,27,10,3$ (529); bereits präzisiert durch C 5, 27, 11, 1-2 (530); VignERON, Politique moralisatrice 583; DERS., Novelle 74.5, 730 f. 
statthaft, wenn eine Ehe bereits im Zeitpunkt der Geburt des Kindes möglich gewesen wäre. ${ }^{27}$

- Die Legitimierung der liberi naturales ist hier auch dann möglich, wenn bereits liberi legitimi aus einer früheren Ehe des Vaters vorhanden sind.

- Einziges anerkanntes Beweismittel für den neuen Ehewillen und die damit entstandene

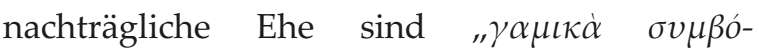
$\lambda \alpha \iota \alpha$ "/,nuptialia instrumenta" - "Eheurkunden".28 - In einer Konstitution von 539 (Nov 89, 1129) wird Justinian außerdem klarstellen, dass ein Kind nicht gegen seinen Willen durch Legitimierung zum gewaltabhängigen und vermögensunfähigen Hauskind unter patria potestas werden kann. Der Gedanke begegnet schon in älteren Quellen. In Nov 74 erscheint er aber nicht, obwohl auch hier deutlich wird, dass die Legitimierung die Kinder zu Hauskindern macht.

\section{Die Legitimierung von liberi naturales durch kaiserliches Reskript auf Antrag des Vaters: Nov 74, 1-2 pr.}

Desweiteren sieht Justinian als eine Neuheit die Legitimierung der liberi naturales durch kaiserliches Reskript auf Antrag des Vaters vor. ${ }^{30}$

Die Voraussetzungen sind:

\footnotetext{
${ }^{27}$ Dazu schon C 5, 27, 11 pr: „eam tamen, cum qua poterat habere conubium".

${ }^{28}$ Nov 74, praefatio, pr, Nov 74, 2; TOMULESCU, Justinien et le concubinat $315 \mathrm{f}$.

${ }^{29}$ Nov 89, 11.

${ }^{30}$ FAYER, Familia romana 3, 49f.; LUCHETTI, Legittimazione 289-321; NIZIOLEK, Legal effects of concubinage 84-89; VAN DE WIEL, Complément à la légitimation 453-465; BONFANTE, Corso 1, 375; JANEAU, Adrogation 91f.; DESPIAU, Enfant né du concubinatus 73-74; MENARD, Concubinat et enfants naturels 55-57; MEYER, Römischer Konkubinat 151f.
}

- Die Ehe der Eltern ist unmöglich. Justinian gibt Beispiele: Die Mutter ist tot; ${ }^{31}$ die Ehe ist aus rechtlichen oder sittlichen Gründen unmöglich;;2 oder (etwas ausgefallen): die Kinder haben die Mutter versteckt. ${ }^{33}$

- Es sind keine liberi legitimi vorhanden. ${ }^{34}$

- Der Vater muss einen persönlichen Antrag,

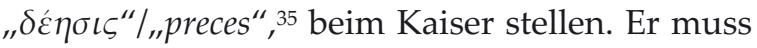
die Gründe für die Unmöglichkeit der Ehe darlegen und den Willen bekunden, die Kinder zu legitimieren und unter seiner patria potestas zu haben. Die Legitimierung beginnt mit diesem Antrag. ${ }^{36}$

- Die Legitimierung durch Reskript ist Hilfe

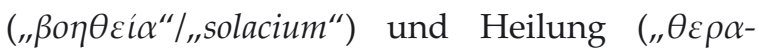
$\pi \varepsilon \dot{\alpha} \alpha$ "/,medela“) ${ }^{37}$ durch den Kaiser.

\section{So genannte Legitimierung von liberi naturales "durch Testament" = kaiserliches Reskript auf Antrag der Kinder: Nov 74, 2, 1}

Das kaiserliche Reskript kann aber auch von den liberi naturales beantragt werden; das ist der Fall der ebenfalls neuen Legitimierung „durch Testament".38

\footnotetext{
${ }^{31}$ Nov 74, praefatio, 1.

${ }^{32}$ Nov 74, praefatio, 1.

${ }^{33}$ Nov 74, praefatio, 2.

${ }^{34}$ Nov 74, 1.

${ }^{35}$ Die Modalität werden in einer Konstitution von Kaiser Zenon genannt, C 1, 23, 7 pr (477): „Universa rescripta, sive in personam precantium [...] si preces veritate nituntur".

${ }^{36}$ Nov 74, 2, pr.

${ }^{37}$ Nov 74, 1-2, pr.

${ }^{38}$ Migliorini, Adozione 364-368; FAYER, Familia romana 3, 50-51; LUCHETTI, Legittimazione 300-303; NIZIOLEK, Legal effects of concubinage 88f.; VAN DE WIEL, Complément à la légitimation 465-468; VOCI, Diritto Ereditario 129f.; JANEAU, Adrogation 120f.;
} 
Dafür ist Voraussetzung ${ }^{39}$ :

- Die Ehe der Eltern war unmöglich.

- Es sind keine liberi legitimi vorhanden.

- Der Vater hat aufgrund zufälliger Umstände

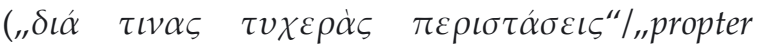
quasdam fortuitas circumstantias") kein Reskript beantragt.

- Im Angesicht des Todes hat der Vater die Kinder testamentarisch als "legitime Erben" (entspr. Hauskindern) eingesetzt. Dazu müssten sie eigentlich liberi legitimi sein.

- Die Kinder haben nach dem Tod des Vaters die Legitimierung durch Reskript beantragt und dabei das Testament und die zufälligen Umstände bewiesen.

- Von der Legitimierung sagt Justinian, sie sei

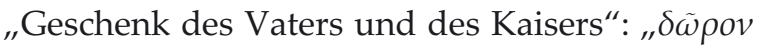
$\tau \alpha \tau \rho o ́ \varsigma ~ \tau \varepsilon ~ \kappa \alpha i ~ \beta \alpha \sigma \iota \lambda \varepsilon ́ \omega \varsigma^{\prime \prime} /$, donum patris et principis."

\section{Ideologie der Novelle 74}

Durch die gesamte Novelle 74 zieht sich eine ideologisch aufgeladene Rhetorik.

Nach Justinian entspricht die von ihm dargestellte Rechtslage den natürlichen Gesetzen

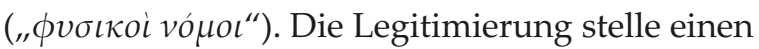
natürlichen Zustand der Kinder wieder her: Nach der Natur seien alle Kinder frei und voll-

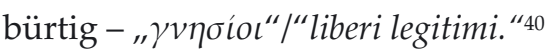

Doch auch die "$\sigma \omega \phi \rho o \sigma v v \eta^{\prime \prime /, c a s t i t a s ", ~ d i e ~}$ Reinheit der Sitten, ist maßgeblich: Nov 74, 4 pr.

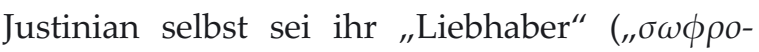
$\sigma u ́ v \eta \varsigma \dot{\varepsilon} \sigma \mu \dot{\varepsilon} v \quad \dot{\varepsilon} \rho \alpha \sigma \tau \alpha i{ }^{\prime \prime} /$,castitatis sumus amatores") und empfehle dasselbe den Untertanen. Aber: "Nichts ist stärker als der Liebeswahn

DESPIAU, Enfant né du concubinatus 74; MEYER, Römischer Konkubinat 152.

${ }^{39}$ Nov 74, 2, 1.

${ }^{40}$ Nov 74, 4, pr.

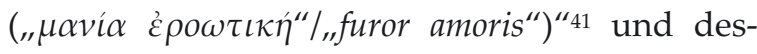
halb bedürfe es der Zügelung durch ein sittenreines Gesetz. Die Reinheit der Sitten sei es auch gewesen, die seinen Vorgänger Justin im Jahr 519 veranlasst habe, frühere Möglichkeiten der Legitimierung durch Adoption/Adrogation ab-

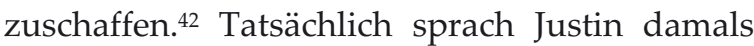
vom Kampf gegen die „iniusta libidinum desideria“, die „unberechtigten Wünsche der Begehren". Zu diesem Kampf bekennt sich auch Justinian. ${ }^{43}$

Schließlich betont Justinian, dass die Legitimierung durch kaiserliches Reskript eine Neuerung ohne Vorläufer sei. Ein „neuer und jetzt gefun-

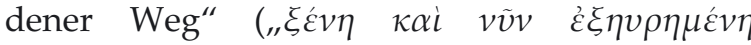

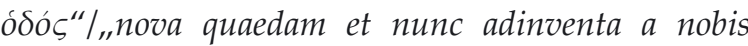
via“), der die bereits bestehenden Möglichkeiten lediglich ergänzen will.44

\section{Verhältnis zur adrogatio}

Aber ist die Legitimierung durch kaiserliches Reskript tatsächlich so neu? Wie verhält sie sich zur abgeschafften adrogatio? ${ }^{45}$ Ein Vergleich der Tatbestände liegt nahe.

- In beiden Fällen kann ein unverheirateter Mann einen filius legitimus erhalten; im Fall des kaiserlichen Reskript dürfen die Eltern nicht verheiratet sein; für die adrogatio ist eine Ehe des Adrogators jedenfalls nicht erforderlich.

- Für die Legitimierung durch Reskript dürfen keine liberi legitimi bereits vorhanden sein; für die adrogatio darf der Adrogator weder vorhandene liberi legitimi noch irgendeine Aussicht auf zukünftige haben.

\footnotetext{
${ }^{41}$ LANATA, Figli della passione 488f.; SANDIROCCO, Concubinato nella antichità $197 \mathrm{f}$.

${ }^{42}$ C 5, 27, 7, 1: "non adrogationum vel adoptionum praetextus quae ulterius minime ferendae sunt."

${ }^{43}$ Nov 74, 3.

${ }^{44}$ Nov 74,1

${ }^{45}$ FAYER, Familia romana 1, 291f. GARDNER, Status, Sentiment and Strategy 64.
} 
- Bei der Legitimierung durch Reskript werden die liberi naturales zu liberi legitimi und kommen unter die patria potestas des Vaters. Bei der adrogatio erhält eine gewaltfreie persona sui iuris die Stellung eines filius legitimus und gerät unter die patria potestas.

- Weder der durch Reskript Legitimierte noch der Adrogierte darf sich dem Umstand widersetzen, dass er zur Person alieni iuris unter patria potestas wird.

- In beiden Fällen muss der Antrag persönlich gestellt werden; einmal vor dem Kaiser, einmal vor der zuständigen Volksversammlung, den Kuriatskomitien.

- Der Eintritt in die väterliche Gewalt ist in beiden Fällen von der Genehmigung einer Autorität abhängig, einmal des Kaisers durch Reskript, einmal der Kuriatskomitien durch adrogatio im engeren Sinne.

Die Legitimierung durch Reskript verläuft also weitgehend parallel zur adrogatio, auch wenn Justinian in unserer Novelle darauf besteht, dass er das Verbot der Legitimierung durch adoptio und adrogatio seines Vorgängers Justin beibehält. ${ }^{46}$

Nicht nur der legitimierende Effekt des kaiserlichen Reskripts entspricht der adrogatio, auch das Verfahren einer "adrogatio per rescriptum principis"47 ist als solches keineswegs neu. Die Hinweise auf eine Praxis der adrogatio durch Reskript gehen weit zurück. Die Entwicklung sei rückläufig skizziert:

a) Die ursprüngliche adrogatio vor den Kuriatskomitien war zur Zeit Justinians in die Zuständigkeit des Kaisers übergegangen. Im Text der Digesten ist die Volksversammlung durch den Kaiser ersetzt, D 1, 7, 2 (Gai 1 inst): "Principis auctoritate adoptamus eos qui sui iuris sunt: Quae species adoptionis dicitur adrogatio." Der Vergleich des Originals der Gaius-Institutionen im

\footnotetext{
${ }^{46}$ Contra NizIOLEK, Legal effects of concubinage $88 \mathrm{f}$.

${ }^{47}$ BONFANTE, Corso 1, 31f.
}

Bereich der adrogatio mit der Fassung desselben Textes in den Digesten zeigt die Veränderung von "populi auctoritate“ $\mathrm{zu}$ "principis auctoritate"; denn Gai. 1,98 lautet: "Populi auctoritate adoptamus eos qui sui iuris sunt: Quae species adoptionis dicitur adrogatio." Das "principis auctoritate" der Digestenüberlieferung ist eine handfeste Interpolation, spätestens (und mit größter Wahrscheinlichkeit) aus der Zeit der justinianischen Kompilation. ${ }^{48}$

b) Schon die Konstitutionen Diokletians vom Ende des dritten Jahrhunderts belegen die adrogatio „ex indulgentia principali / ex rescripto principali" als geleichwertigen Ersatz für die adrogatio "per populum iure antiquo" nach altem Recht vor der Volksversammlung, C 8, 47, 2, 1.49

c) Die Verlagerung der adrogatio von den Kuriatskomitien auf den Kaiser ist dabei im Zusammenhang mit dem Amt des pontifex maximus als Teil der römischen Kaiserwürde $\mathrm{zu}$ sehen; nach republikanischen Vorstellungen würde ihm daher der Vorsitz in den Kuriatskomitien gebühren und damit die Verfahrensleitung der adrogatio.

d) Dass Kinder, die vor der (kaiserlichen) Verleihung des römischen Bürgerrechts an ihren Vater (samt seinen Kindern) geboren waren, durch Kaiserkonstitution der patria potestas zugeführt werden, also der Lage römischer liberi legitimi, berichtet Gaius unter Hinweis auf ein edictum Hadrians, Gai. 1, 93.50 Auch wenn das

\footnotetext{
${ }^{48}$ Kunst, Adoption und Testamentsadoption 89; FAYER, Familia romana 1, 303f.; BONFANTE, Corso 1, $32 \mathrm{f}$.

${ }^{49}$ C 8, 47, 2, 1 (286): "ac si per populum iure antiquo facta esset"; GARDNER, Status, Sentiment and Strategy 70; BONFANTE, Corso 1, 32, mit weiteren Beispielen.

${ }^{50}$ Gai. 1, 93 "Si peregrinus sibi liberisque suis civitatem romanam petierit, non aliter filii in potestate eius fiunt, quam si imperator eos in potestatem redegerit. Quod ita demum is facit, si causa cognita aestimaverit hoc filiis expedire; diligentius autem exactiusque causam cognoscit de impuberibus absentibusque: et haec ita edicto divi hadriani significantur."
} 
Wort adrogatio hier keine Verwendung findet, handelt es sich in der Sache doch um den Wechsel einer gewaltfreien Person in die patria potestas und damit im Ergebnis um eine "adrogatio per rescriptum principi".

Ziehen wir den Kreis über die Strukturen der adrogatio hinaus, so finden sich immer neue Varianten der Legitimierung. Zwei Beispiele für eine Legitimierung "per rescriptum principis" $u$. ä. aus der Zeit vor Justinian seien genannt:

- Aus der zweiten Hälfte des zweiten nachchristlichen Jahrhunderts, in D 23, 2, 57, stammt ein kaiserliches Reskript an eine Frau - Flavia Tertulla -, die mehrere Kinder (numero liberorum vestrorum) aus der jahrzehntelangen Verbindung (in eo matrimonio quaesitorum, quod ante annos quadraginta contractum est) mit ihrem Onkel hat. Die Frage ist nicht, ob man diese Verbindung legitimieren kann: Sie ist verboten ${ }^{51}$ und kann keine Bestätigung finden. ${ }^{52}$ Es geht allein um den Status der Kinder; dort greifen die Kaiser zu einer Fiktion: Die Kinder sind so anzusehen, als „wären sie in gesetzlicher Weise empfangen worden" ("perinde atque si legitime concepti fuissent"). Von einem Eintritt der Kinder in die patria potestas und einer adrogatio ist keine Rede zumal der Vater inzwischen wohl verstorbenen ist (das Perfekt fuisti findet Verwendung). Um eine Legitimierung durch Reskript handelt es sich dennoch.

- Das "senatus consultum de Campanis" von 188 v. Chr.53 erklärt die bereits vorhandenen Kinder von (nicht römischen) Kampanern und römischen Frauen $\mathrm{zu}$ "iusti liberi heredesque“. Es handelt sich nicht um römische Bürger; die Kin-

\footnotetext{
${ }^{51}$ Gai. 1, 62: "Sororis vero filiam uxorem ducere non licet."

52 D 48, 5, 39, 6 (Pap. 36 quaest.): "Incestae nuptiae confirmari non solent."

${ }^{53}$ Liv. 38, 36: "Campani [...] petierunt ut sibi ciues Romanas ducere uxores liceret, et si qui prius duxissent, ut habere eas, et nati ante eam diem uti iusti sibi liberi heredes que essent, utraque res impetrata."
}

der kommen nicht unter die römische patria potestas. Von adrogatio kann daher keine Rede sein, wohl aber von „Legitimierung durch Senatsbeschluss"; das senatus consultum lässt sich als einer der normativen Vorläufer der Kaiserkonstitution inklusive Reskript verstehen.

Diese Beispiele zeigen, dass die Produktion von liberi legitimi, die Verwandlung von gewaltfreien Personen in (römische) Hauskinder, die Technik einer adrogatio oder Legitimierung "per rescriptum principis" nicht neu und keine Erfindung Justinians sind ${ }^{54}$. Das Neue bei Justinian beschränkt sich weitgehend auf die Vermeidung der Bezeichnung „adrogatio“.

\section{Verhältnis zur adrogatio postuma}

Wenden wir uns zum Schluss nochmals Justinians Legitimierung durch Testament zu. Nach dem Befund bei den Reskripten an Lebende drängt sich auch hier die Frage nach möglichen Vorläufern auf.

Hier liegt ein Vergleich mit der Adoption durch Testament (adoptio per testamentum ${ }^{55}$ ) nahe, im Fall einer adoptierten Person sui iuris handelt es sich wiederum um eine adrogatio; hier spricht man gerade von einer adrogatio postuma.

- In beiden Fällen kann ein unverheirateter Mann einen filius legitimus erhalten; im Fall des kaiserlichen Reskript dürfen die Eltern nicht

\footnotetext{
54 BONFANTE, Corso 1, 375.

${ }^{55}$ CHEVReAU, Le temps 244f.; GARDNER, Status, Sentiment and Strategy 63-79; KUNST, Adoption und Testamentsadoption 87-104; KURYLOWICZ, Nachklassische Adoption 187-201; FAYER, Familia romana 1, 351-361; GAUDEMET, Formes et fonctions de l'adoption 275-300; AMELOTTI, Testamento romano 1, 153f.; Bonfante, Corso 1, 26-29; Albanese, Persone 236-238; VOLTERRA, Adozione testamentaria 469f.; PREVOST Adoptions politiques 30f.; GONNET, Droit du magistrat 235-237; CUQ, Institutions 2, 202; LEFAS, Adoption testamentaire 721f.
} 
verheiratet sein; für die adrogatio postuma ist eine Ehe des Adrogators jedenfalls nicht erforderlich. - Die Legitimierung durch Testament ist nur möglich, wenn der Vater die Legitimierung durch kaiserliches Reskript beantragen wollte, es aber aufgrund "zufälliger Umstände" nicht dazu gekommen ist; das heißt, die Legitimierung durch Testament steht unter sehr engen Voraussetzungen. Die adrogatio postuma hingegen ist ein außerordentliches Mittel, dem Antragsteller eine adrogatio $\mathrm{zu}$ ermöglichen, auch wenn sie unter Lebenden nicht möglich wäre. Sie hat also besonders weite Voraussetzungen.

- In beiden Fällen dürfen keine liberi legitimi bereits vorhanden sein.

- In beiden Fällen muss der Antrag von den Kindern beziehungsweise Adrogierten persönlich gestellt werden.

- Der Eintritt in die väterliche Gewalt ist in beiden Fällen von der Genehmigung einer Autorität abhängig, einmal des Kaisers durch Reskript, einmal der Kuriatskomitien, oder des Prätors; aber auch bei der adrogatio per testamentum kommt eine Genehmigung durch kaiserliches Reskript in Betracht. Eine unwirksame adrogatio kann, wie Marcellus in D 1, 7, 38 ganz allgemein konstatiert, durch kaiserliches Reskript bestätigt werden: "Adoptio non iure facta a principe confirmari potest". Das muss auch für eine adrogatio postuma gelten: Zum Zeitpunkt des Todes ist sie unwirksam.

Die Legitimierung durch Testament verläuft also weitgehend parallel zur adrogatio per testamentum. Das ist ebenso wenig ein „neuer und jetzt gefundener Weg" wie die Legitimierung durch kaiserliches Reskript.

Eines der berühmtesten Beispiele für eine testamentarische adrogatio finden wir am Ende der römischen Republik: Die Adoption des C. Octavius/Octavianus durch seinen Großonkel
C. Iulius Caesar. ${ }^{56}$ Caesar wurde am 15. März 44 v. Chr. ermordet. Seit 8. Mai 44 v. Chr. trug Octavius den Namen C. (Iulius) Caesar. ${ }^{57}$ Vom letzten Testament des Diktators ${ }^{58}$ sagt Sueton, ${ }^{59}$ Caesar habe dort seinen Großneffen nicht nur zum Erben eingesetzt, sondern auch "in familiam nomenque“ adoptiert - „in die (männliche) Familie und den Namen". Octavius war zum Zeitpunkt der Ermordung Caesars sui iuris ${ }^{60}$; insofern konnte er nur „,adrogiert" werden.

Zur "Adoption in die Familie“ Caesars berichten die Geschichtsschreiber Appian und Cassius Dio, ${ }^{61}$ dass Octavius aufgrund der enormen Bedeutung dieser Adoption die Genehmigung durch die Kuriatskomitien anstrebte und dabei

\footnotetext{
${ }^{56}$ Dazu und dagegen: KUNST, Adoption und Testamentsadoption 95f.; CHEvreau, Le temps 244f.; SCHUMACHER, Oktavian 49f.; FAYER, Familia romana 1, 351f.; RUGGERI Datio 1, 153f.; SCHMITTHENNER, Oktavian 39f.; VocI, Diritto Ereditario 130; AMELOTTI, Testamento romano 1, 12f. und 152f.; BONFANTE, Corso 1, 26f.; Albanese, Persone 236-238; VOlterRa, Adozione testamentaria 474f.; PAOLI, Testament 529f.; HENNE, A propos 141f.; LeMOSSE, Adoption d'Octave 371f.; PrEVOST, Adoption d'Octave, 361f.; DERS. Adoptions politiques 29f., 68f.; SIBER, Entwicklung 26f.; JANEAU, Adrogation 121-122.

${ }^{57}$ KIENAST, Römische Kaisertabelle 61, 67. Dazu CIL 5, 4305 = ILS 75; FAYER, Familia romana 1, 357.

${ }^{58}$ Testament vom 13. 9. 45 v. Chr.; SCHUMACHER, Oktavian 49f.; PAOLI, Testament 546; PreVOST, Adoptions politiques 29; SCHMITTHENNER, Oktavian $13 f$.

${ }^{59}$ Suet. Iul. 83, 2-3: "In ima cera Caium Octavium etiam in familiam nomenque adoptavit [...]", Suet. Aug. 7, 2: "Postea Cai Caesaris et deinde Augusti cognomen assumpsit, alterum testamento maioris avunculi". Auch Vell. 2, 59, 1: "Caesaris deinde testamentum apertum est, quo C. Octauium, nepotem sororis suae Iuliae, adoptabat"; FAYER, Familia romana 1, 355 Anm. 267; RUGGERI Datio 1, 153f.; VolterRA, Adozione testamentaria $470 \mathrm{f}$.

${ }^{60}$ Suet. Aug. 4, 1; 8, 1. SCHUMACHER, Oktavian 49 f.

${ }^{61}$ App. civ. 3, 14, 94, und 3, 1, 11; Cass. Dio 44, 35, 2-3; 45, 5, 3 und 46, 47, 4f.; Kunst, Adoption und Testamentsadoption 99f.; FAYER, Familia romana 1, 355f.; Lemosse, Adoption d'Octave 371f.; VOLTERRA, Adozione testamentaria 474f.; SIBER, Entwicklung 26f.; GONNET, Droit du magistrat 236f.
} 
auf den Widerstand des M. Antonius stieß. Noch vor Promulgation der lex curiata begab sich Octavius, so berichtet Appian, mit Zeugen zum Prätor, ${ }^{62}$ um seine Annahme der testamentarischen Adoption zu erklären und die Adoption ratifizieren zu lassen. $\mathrm{Ob}$ damit die Rolle des Prätors richtig erfasst ist, ist nicht unzweifelhaft. Später kam es aber auch zum Beschluss der Kuriatskomitien.

Octavius erlangte durch Testament und Bestätigung durch den Hoheitsträger (spätestens durch die Kuriatskomitien) die Position eines legitimen Sohn Caesars: eine „adrogatio postuma“.

Von unserer Exkursion in die späte Republik zurück zu Justinian: Mit seinem Regelungsproblem der Legitimierung von liberi naturales und seinen Methoden der Legitimierung durch Reskript und durch Testament steht Justinian entgegen seiner Ideologie der gesetzgeberischen Neuheiten - jeweils in einer langen Tradition.63 Wirklich neu an seiner Gesetzgebung in Novelle 74 ist aber der Versuch eines Systems der Legitimierungsarten für liberi naturales, deren Status präzise erfasst wird.

\section{Korrespondenz:}

Sandrine Vallar

Löwengasse 38/8, 1030 Wien, Austria

sandrinevallar@hotmail.fr

\footnotetext{
${ }^{62}$ App. bell. civ. 3, 1, 14. PAOLI, Testament 529f.

${ }^{63}$ Dazu JaneaU, De l'adrogation 114f.
}

\section{Abkürzungen:}

$\begin{array}{ll}\text { App. } & \text { Appianus, Bella civilia } \\ \text { Cass. Dio } & \text { Cassius Dio } \\ \text { CIL } & \text { Corpus Inscriptionum Latinarum } \\ \text { Gai. } & \text { Gaius, Insitutiones } \\ \text { ILS } & \text { Inscriptiones Latinae Selectae } \\ \text { Liv. } & \text { Livius, Ab urbe condita } \\ \text { NRHD } & \text { Nouvelle revue historique de droit } \\ & \text { français et étranger } \\ \text { Nov } & \text { Novellae } \\ \text { Paul. sent. } & \text { Iulius Paulus, Sententiae } \\ \text { Plaut. } & \text { Plautus } \\ \text { RHD } & \text { Revue historique de droit français et } \\ & \text { étranger } \\ \text { RIDA } & \text { Revue Internationale des Droits de } \\ & \text { l'Antiquité } \\ \text { SDHI } & \text { Studia et Documenta } \\ \text { Suet. Aug. } & \text { Historiae et Iuris } \\ \text { Suet. Iul. } & \text { Suetonius, Divus Augustus } \\ \text { TR } & \text { Suetonius, Divus Iulius } \\ \text { Vell. } & \text { Tijdschrift voor Rechtsgeschiedenis } \\ & \text { Velleius Paterculus, } \\ & \text { Historiae Romanae }\end{array}$

\section{Literatur:}

Bernardo AlBANESE, Le persone nel diritto privato romano (Palermo 1979).

Mario AMELOTTI, Il testamento romano attraverso la prassi documentale, Bd. 1: Le forme classiche di testamento (Firenze 1966).

Pietro Bonfante, Corso di diritto romano, Bd. I: Diritto di famiglia (Milano ${ }^{2}$ 1963).

Luigi CAPOGROSSI COLOGNESI, Tollere liberos: un mito dei moderni? in: Holger AltMePPEN u.a. (Hgg.), FS für Rolf Knütel (Heidelberg 2009) 131-146.

Emmanuelle Chevreau, Le temps et le droit: La réponse de Rome (Paris 2006).

Rodolfo DANIELI, Sul concubinato in diritto giustinianeo, in: Studi in onore di Vincenzo Arangio-Ruiz nel XLV anno del suo insegnamento, Bd. III (Milano 1953) 175-179.

Henri DESPIAU, Droit romain: De l'enfant né du concubinatus (Thèse Toulouse 1885).

Carla FAYER, La familia romana, Aspetti giuridici ed antiquari, Prima parte (Roma 1994).

Carla FAYER, La familia romana, Aspetti giuridici ed antiquari. Concubinato divorzio adulterio, Parte terza (Roma 2005). 
Raimund FRIEDL, Der Konkubinat im kaiserzeitlichen Rom (Stuttgart 1996).

Jane GARDNER, Status, Sentiment and Strategy in Roman Adoption, in: Adoption et Fosterage (Paris 1999) 63-79.

Jean GAUDEMET, Droit romain et principes canoniques en matière de mariage au bas Empire, in: Studi Emilio Albertario (Milano 1953) 173-196.

Jean GAUDEMET, Formes et fonctions de l'adoption dans le monde antique, in: Droit et société aux derniers siècles de l'empire romain (Naples 1992) 275-300.

Jean GAUDEMET, Justum matrimonium, in: RIDA 3 (1949) 309-366.

Jean GAUDEMET, Union libre et mariage dans la Rome impériale, in: Le droit de la famille en Europe son évolution depuis l'Antiquité jusqu'à nos jours, Actes des Journées Internationales d'Histoire du Droit publiés sous la direction de Ganghofer R. (Strasbourg 1992) 377-392.

Paul GONNET, Le droit du magistrat romain, in: Rhd 16 (1937) 235-237.

Henri Henne, A propos du testament de César, in: Mélanges H. Lévy-Bruhl (Paris 1959) 141-151.

Hubert JANEAU, De l'adrogation des liberi naturales à la légitimation par rescrit du Prince (Paris 1947).

Max Kaser, Das Römische Privatrecht, 2. Abschnitt : Die nachklassischen Entwicklungen (München 21975).

Evanghelos C. KARABELIAS, La pratique du concubinat avec une femme libre, affranchie ou esclave dans le droit post-classique, in: Atti dell'Accademia Romanistica Costantiniana, VII Convegno Internazionale (Perugia 1985) 183-201.

Dietmar KIENAST, Römische Kaisertabelle. Grundzüge einer römischen Kaiserchronologie (Darmstadt 21996).

Christiane KUNST, Adoption und Testamentsadoption in der Späten Republik, in: Klio 78 (1996) 87-104.

Marek KurYLOWICZ, Zur nachklassischen Adoption, in: Ars boni et aequi. FS für Wolfgang Waldstein (Stuttgart 1993) 187-201.

Giuliana LANATA, I figli della passione. Appunti sulla novella 74 di Giustiniano, in: Atti dell'Accademia Romanistica Costantiniana, VII Convegno Internazionale (Perugia 1985) 487-493.

Edouard LE DeufF, Du Concubinatus (Thèse Rennes 1893).

Alexandre LEFAS, L'adoption testamentaire à Rome, in: Nrhd 21 (1897) 721-763.

Bernardo LeMOSSE, L'adoption d'Octave et ses rapports avec les règles traditionnelles du droit civil, in: Studi in onore di Albertario E. (Milano 1943) 371-395.

Giovanni LUCHETTI, La legittimazione dei figli naturali nelle fonti tardo imperiali e Giustinianee (Milano 1990).

Eugène MENARD, Concubinat et des enfants naturels, Thèse (Poitiers 1882).

Paul MEYER, Der römische Konkubinat nach den Rechtsquellen und den Inschriften (Leipzig 1895).

Marco MigLIORINI, L'adozione tra prassi documentale e legislazione imperiale nel diritto del tardo impero romano (Milano 2001)

Marian NIZIOLEK, Legal effects of concubinage in reference to concubine's offspring in the light of imperial legislation of the period of dominate (Warsaw-Kraków 1980).

Marian NizIOLEK, Meaning of the Phrase liberi naturales in Roman Law Sources up to Constantine's reign, in: Rida 22 (1975) 317-344.

Lise Arends OlsEN, La femme et l'enfant dans les unions illégitimes à Rome, L'évolution du droit jusqu'au début de l'Empire (Bern 1999).

Jules PAOLI, Le testament calatis comitiis et l'adrogation d'Octave, in: Studi in onore di E. Betti, Bd. 3 (Milano 1962) 529-563.

Leo Peppe, Paelex e spurius, in: Mélanges à la mémoire de André Magdelain (Paris 1998) 343-359.

Jean PlASSARD, Le concubinat romain sous le haut empire, Thèse (Toulouse 1921).

Alfredo Mordechai RABELLO, Il problema dei matrimoni fra ebrei e cristiani nelle legislazione imperiale e in quella della chiesa (IV-VI secolo), in: Atti dell'Accademia Romanistica Costantiniana, VII Convegno Internazionale (Perugia 1985) 213-224.

Marcel-Henri PREVOST, L'adoption d'Octave, in: Rida 4 (1950) 361-381.

Marcel-Henri PREVOST, Les adoptions politiques à Rome sous la République et le Principat (Paris 1949).

Aline Rousselle, Concubinat et adultère, in: Opus 3, International journal for social and economic history of Antiquity 3 (1984/1) 75-84.

Carmela Russo RuggerI, La datio in adoptionem, Bd. 1 (Milano 1990).

Eleonora SANDIROCCO, Il concubinato nella antichità tra legge laica e visione religiosa, in: Labeo 50 (Napoli 2004) 197-230.

Nicola SANTORO, Sul 'Tollere liberos', in: Index 28 (2000) 273-278.

Walter SCHMITTHENNER, Oktavian und das Testament des Cäsars. Eine Untersuchung zu den politischen Anfängen des Augustus (München 1952) 
Leonhard SCHUMACHER, Oktavian und das Testament Caesars, in: ZRG RA 116 (1999) 49-70.

Heinrich SIBER, Zur Entwicklung der römischen Prinzipatsverfassung (Leipzig 1933).

Siro SolAzZI, Il concubinato con l'obscuro loco nata, in: Sdhi 13/14 (1947/1948) 269-277.

Constantin St. TOMULESCU, Justinien et le concubinat, in: Studi Scherillo (Naples 1972) 299-326.

Jacob URBANIK, A broken marriage promise and Justinian as lover of chastity, on Novela 74 and P. Cair. Masp. I 67092 (Ad 553), in: The Journal of Juristic Papyrology 41 (2011) 123-151.

C. VAN DE WIEL, La légitimation par mariage subséquent, de Constantin à Justinien. Sa réception sporadique dans le droit byzantin, in: Rida 25 (1978) 307-350.

C. VAN DE WIEL, Complément à la légitimation par mariage subséquent sous Justinien et dans le droit gréco-romain, in: Rida 26 (1979) 453-465.

Roger VIGNERON, La politique moralisatrice de Justinien à l'égard des séducteurs au vu de la Nov 74.5, in: Collation iuris romani, FS Hans Ankum, Bd. II (Amsterdam 1995) 583-592.

Roger VignerOn, La Novelle 74.5 de Justinien et le régime du concubinat romain, in: R. GANGHOFER, Le droit de la famille en Europe son évolution de- puis l'Antiquité jusqu'à nos jours, Actes des Journées Internationales d'Histoire du Droit publiés sous la direction de. (Strasbourg 1992) 729-737.

Pasquale VocI, Diritto Ereditario Romano I (Milano $\left.{ }^{1} 1960\right)$.

Pasquale VocI, Novi studi sulla legislazione romano del tardo impero (Padova 1989).

Edoardo VOLTERRA, L'Adozione testamentaria ed un 'iscrizione latina e neopunica della Tripolitania, in: DERS., Scritti Giuridici, Bd. VII (Napoli 1999) 469482.

Edoardo VOLTERRA, Iniustum Matrimonium, in: DERS., Scritti Giuridici, Bd. III: Famiglia e Successioni, (Naples 1991) 441-470.

Laurent WAELKENS, Dans quelles limites peut-on parler d'enfants naturels dans le droit romain de l'Antiquité?, in: TR 80 (2012) 399-414.

Alan WAtson, The Law of Persons in The Later Roman Republic (Oxford 1967).

Hans Julius WoLfF, Doctrinal Trends in Postclassical Roman Marriage Law, in: ZRG RA 67 (1950) 261319.

Hans Julius WoLfF, Written and Unwritten Marriages in Hellenistic and Postclassical Roman Law (Haverford 1939). 